\title{
New Approach to Generation and Amplification of the THz Radiation in Plasma Created by Intense Two-Color Laser Fields
}

\author{
$\underline{\text { A. M. Popov }}^{1,2,3}$, A. V. Bogatskaya ${ }^{1,3}$ \\ ${ }^{1}$ D. V. Skobeltsyn Institute of Nuclear Physics, Moscow State University, Moscow, Russia, alexander.m.popov@ gmail.com \\ ${ }^{2}$ Department of Physics, Moscow State University, 119991, Moscow, Russia \\ ${ }^{3}$ P. N. Lebedev Physical Institute, RAS, Moscow Russia
}

Construction of new types of $\mathrm{THz}$ radiation sources is nowadays of sufficient fundamental and practical interest. This interest is motivated by the prospects of using $\mathrm{THz}$ radiation for $\mathrm{THz}$ imaging in a lot applications in science and technology [1]. However, till now $\mathrm{THz}$ frequency band is still insufficiently studied in comparison with infrared and microwave ones. Among others, $\mathrm{THz}$ generators based on the gas ionization by high-intensity femtosecond lasers are widely studied now. Such a way of $\mathrm{THz}$ generation allows to obtain radiation with a continuous spectrum throughout the terahertz bandwidth that is of importance for different spectroscopy applications. Typically, optical-to- $\mathrm{THz}$ conversion results from the four wave mixing process (FWM) in high-intensity femtosecond two color laser field $(\omega+2 \omega)$. Unfortunately, the efficiency of such conversion remains rather small - about $0.001-0.01 \%$. In this respect, problems of amplification of $\mathrm{THz}$ pulses as well as the development of new more efficient methods of optical to terahertz energy conversion are of significant importance. In this paper we propose an alternative way to enhance significantly the process of optical-to- $\mathrm{THz}$ conversion by using the resonance in four-wave mixing process. Such resonant enhancement is possible in the aluminum vapor irradiated by Ti-Sa laser pulse and its second harmonic via the aluminum atom excited state $4 s\left({ }^{2} S_{1 / 2}\right)$ with the energy $E_{4 s} \approx 3.14 \mathrm{eV}$ close to the frequency of the Ti-Sa second harmonic $\hbar(2 \omega) \approx 3.1 \mathrm{eV}$. Schematically this process is shown at fig. $1 \mathrm{We}$ demonstrate that the $\mathrm{THz}$ output can be increased up to two orders of magnitude in comparison with the non-resonant process.

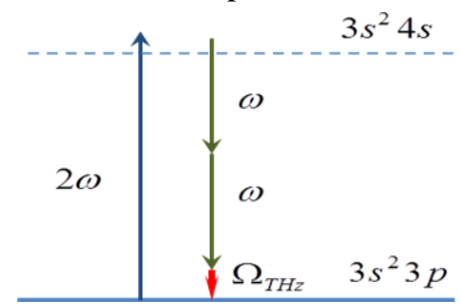

Fig 1. Nearly resonance four-wave-mixing process in aluminum atom

It is known that irradiation of gas by intense two color $(\omega+2 \omega)$ laser pulse results in low-frequency polarization of the medium $P_{\Omega} \sim \chi^{(3)} E_{\omega}^{2} E_{2 \omega}$ and $\mathrm{THz}$ emission [2] which arises from the four-wave mixing (FWM) process $\left(\Omega=\omega^{\prime}+\omega^{\prime \prime}-2 \omega^{\prime \prime \prime}\right)$ and in the limit of perturbation theory is governed by the cubic susceptibility $\chi^{(3)}\left(\omega^{\prime}+\omega^{\prime \prime}-2 \omega^{\prime \prime}\right) \quad\left(\omega^{\prime}, \omega^{\prime \prime}\right.$ are the frequencies from the spectral width of the pulse with frequency $\omega$ while $\omega^{\prime \prime \prime}$ is the frequency from the spectral width of second harmonic $2 \omega$.). For femtosecond laser pulses this low-frequency spontaneous emission appears in the $\mathrm{THz}$ and far IR frequency band. In this paper we plan to study low frequency spontaneous background from gas or plasma irradiated by two-color laser field which could be further amplified according to the mechanism proposed in [3].

Below we analyze the resonant FWM process and the formation of the spontaneous $\mathrm{THz}$ background for xenon and aluminum atoms using the approach developed in [4] that is based on the accurate consideration of quantum system interaction with vacuum quantized field modes in the first order of perturbation theory while the intense laser field is considered classically beyond the perturbation theory.

The electric field strength in the two-color pulse was chosen in a form

$$
E(t)=f(t) \times\left(E_{\omega} \cos (\omega t)+E_{2 \omega} \cos (2 \omega t)\right),
$$

where $\omega$ the frequency of the Ti-Sa laser and $f(t)$ is the envelope being the same for both pulses. It was chosen in the sine-squared form with the half-duration $\tau=880$ atomic units ( $20 \mathrm{fs}$ ), that corresponds to 8 optical cycles of the Ti-Sa laser or 16 optical cycles for its second harmonic.

The study of atomic emission was performed in the range of intensities for both fundamental and second harmonic $\sim 10^{-5}-2 \times 10^{-3}$ at. un. and the intensity of second harmonic is assumed to be several time less than the intensity of the fundamental one. On the one hand, the increment of the intensity gives rise to more efficient FWM process, on the other hand the ionization and the depletion of the ground state will reduce the probability of the resonance FWM.

Typical spontaneous emission spectra obtained in our simulations for aluminum atom are presented at figure 2. Curves 1, 2 and 3 represent the spectra obtained for Ti-Sa laser irradiation, its second harmonic as well as combined two-color action respectively. First of all, the peaks of Rayleigh scattering at $\hbar \omega_{k \lambda}=1.55 \mathrm{eV}$ for fundamental frequency and $\hbar \omega_{k \lambda}=3.1 \mathrm{eV}$ for the second harmonic are observed. The last peak has the triple structure (triplet Mollow) due to the near-resonant transition to $4 \mathrm{~s}$ state. There are also some additional peaks appearing for the Ti-Sa laser resulting from different processes. Most important to highlight that for two-color laser pulse one more low-frequency peak with a maximum at $\hbar \Omega_{k \lambda} \approx 0.2 \mathrm{eV}$ is well pronounced. This peak manifests the FWM process when the second harmonic 
quantum absorption and two quanta of Ti-Sa laser stimulated emission is accomplished by spontaneous emission of $\mathrm{THz}$ - far IR quantum (see figure 1).

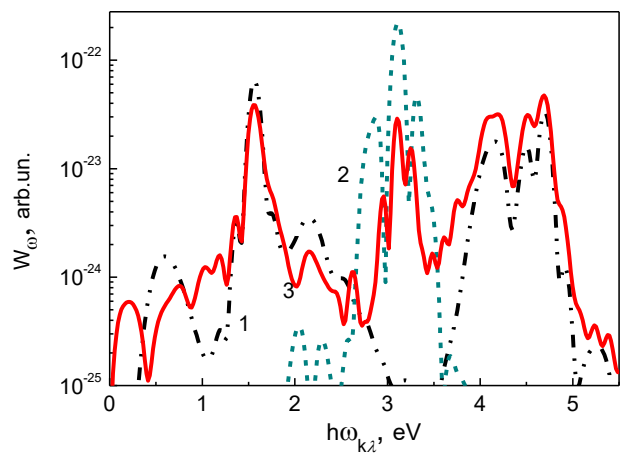

Fig. 2. Spectrum of spontaneous emission of aluminum atom irradiated by Ti-Sa laser pulse with $I_{\omega}=1.4 \times 10^{-3}$ (in atomic units) (1), its second harmonic with $I_{2 \omega}=10^{-4}$ (2) and by two-colour pulse (3) with $I_{\omega}=1.4 \times 10^{-3}$ and $I_{2 \omega}=10^{-4}$.

The detailed analysis of the low-frequency emission in the near resonant FWM process leads to the conclusion that optimal values for $\mathrm{THz}$ emission exist near the intensities $I_{\omega}=1.4 \times 10^{-3}$ and $I_{2 \omega}=4 \times 10^{-4}$.

The resonant character of FWM can dramatically increase $\mathrm{THz}$ emission in comparison with the nonresonant case. To demonstrate it, the simulation of $\mathrm{THz}$ background from the xenon atom was also analyzed for nearly the same laser radiation parameters [5]. Comparative analysis of these data leads to the conclusion that resonant character of FWM provides the enhancement of the emission in $\mathrm{THz}$ bandwidth up to two orders of value.

Further amplification of a seed low-frequency pulse can be realized in the nonequilibrium plasma channel formed independently by the UV laser pulse. Earlier it was demonstrated in [6] that such a plasma produced in the process of multiphoton ionization of heavy rare gases by powerful UV laser pulse is characterized by population inversion that can be used for amplification of THz-band radiation. This amplification is possible if the energy of photoelectron peak is located in the interval of increasing with energy (faster than the linear dependence) electron transport cross section.
Possible realization of such a concept is performed at figure 3. Terahertz spontaneous signal emerges in the region of intense two-color laser action and then propagates through the amplifying zone which is formed by $\mathrm{KrF}$ laser pulse. In the frames of our concept the presence of two different lasers is mandatory because we require high-intensities for the two-color pulse to reach high level of nonlinearity for FWM process but at the same time we do not need high level of ionization in the amplifying plasma channel as domination of electron-electron collisions will cause the fast relaxation of the nonequelibrium electron energy distribution function (EEDF) and destroy the population inversion.

To conclude, in this paper we demonstrate that the resonance between quanta of the laser radiation and energy transition from the ground to one of the excited states of the atomic system can result in the dramatic enhancement of the four-wave mixing process in two-colour laser field $(\omega+2 \omega)$ providing more efficient optical to $\mathrm{THz}$ conversion scheme. Possibility of further amplification of the THz background is discussed. Also, the new concept allowing to enhance the $\mathrm{THz}$ output from the two-color scheme in a nonequilibrium plasma channel formed by UV laser was proposed.

This work was supported by the Russian Science Foundation (project no. 18-72- 00125).

\section{References}

1. Mittleman, D. M. Twenty years of terahertz imaging // Opt. Express 2018.V. 26, P. 9417

2. Cook, D. J. and Hochstrasser, R. M. Intense terahertz pulses by four-wave rectification in air // Opt. Lett. 2000. V. 25, P. 1210

3. Bogatskaya, A. V., Volkova, E. A., Popov, A. M. Generation and amplification of sub- $\mathrm{THz}$ radiation in a plasma of rare gases formed by two-color femtosecond laser pulse // Las. Phys. Lett. 2018. V. 15, P. 065301

4. Bogatskaya, A. V., Volkova, E. A., Popov, A. M. Spontaneous transitions in atomic system in the presence of high intensity laser field // Europhysics Letters 2016. V. 116, P. 14003

5. Bogatskaya, A. V., Popov, A. M. New approach to the problem of $\mathrm{THz}$ generation by intense two-color laser fields // Laser Phys. 2018. V. 28 submitted

6. Bogatskaya, A. V., Popov, A. M. On the possibility of the amplification of subterahertz electromagnetic radiation in a plasma channel created by a high-intensity ultrashort laser pulse // JETP Lett. 2013. V. 37. P. 388-392

amplified $\mathrm{THz}$ pulse

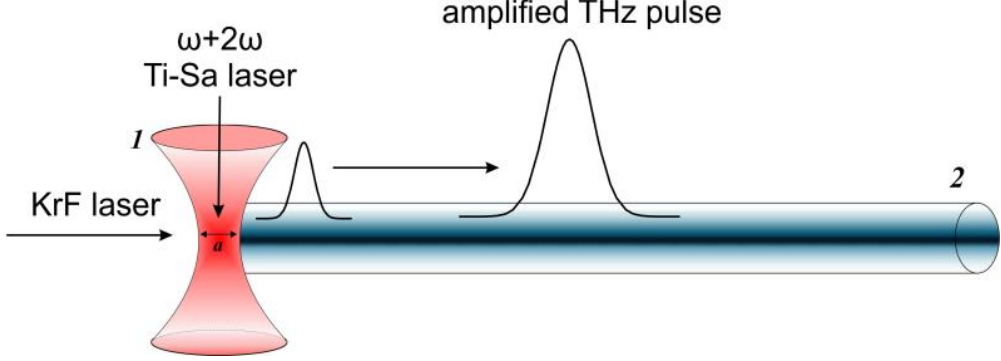

Fig. 3. Principal scheme of THz pulse generation and amplification in case of perpendicular geometry. THz signal is produced in the region of focal waist of two-color Ti-Sa laser action (channel 1) and then enhanced in the 2nd channel formed in xenon by KrF laser pulse. 